\title{
What can optical engineers learn from Leonardo?
}

\author{
I.L. Livshits1 and O.Faehnle2 \\ 1 ITMO University, Saint Petersburg, 197101, Russian Federation \\ 2 OST-University of Applied Sciences, Buchs, 9471, Switzerland)
}

\begin{abstract}
Optics in general is an old science. Many brilliant researchers have created both theory and practice in this field. Some of their achievements are still in use, some are unjustly forgotten. In this paper we tried to rethink some points of ancient scientists to show that they had «seeds» which are still important ourdays. In particular, the applicability of the "golden section" from nature to the modern, computational process of optical system design was tested experimentally. Design principles, current challenges, possible solutions and some applications are discussed. They could be used, for example, in optical design, at the stage of starting scheme or help making decision of optical system producibility.
\end{abstract}

\section{Indroduction}

State-of-the-art optical technologies are characterized by the highest levels of "precision" possible today. At the same time, the step towards optical applications of tomorrow (e.g. for telescopes, endoscopes, lithography machines or lidar systems) requires the synthesis of know-how of a wide variety of technology fields, such as materials science, optical surface generation, metrology and chemistry as well as mechanical engineering and software development. Consequently, it is essential to develope and apply innovative research methods that are capable of enabling such syntheses [1-4]. Following that trail, the analysis of the morphology of the word re-search itself might be of help. We find it to be a combination of two morphemes: the latin words "re" (meaning "back") and "circare" (meaning "go about"). If we now apply both parts literally and travel back in time to mankind's origins and start searching there for multi-disciplinary solutions, we find ourselves observing nature herself. Doing so, mankind converted already several solutions from nature into high end technology applications in industry: e.g, gecko's feet's van-der-Waals' contact while walking up walls has been applied to bonding components; chamaeleon's gentle grip applied to industrial robots handling delicate parts or, last not least, butterfly's beautifully colored wings inspiring us to developing diffractive optical elements.

One omnipresent effect in nature is the golden ratio (1.618), firstly described by Euclid [5]. It describes the shape of nautilus shells, hurricans and galaxies as well as the hunting pattern of hawks. In general, it seems to be often the golden solution to solve multitrandisciplinary and complex situations in nature. Therefore we assume the golden ratio a matter, worthwhile analyzing even from the perspective of leveling-up "precision" towards tomorrows optical technology solutions. This paper reports on authors first curious steps analyzing the impact of natures golden ratio, phi, onto high end optical systems design. When we look back at historical times of mankind and if we are interested in optics, our attention is attracted by the time of the renaissance epoch where brilliant and gifted people worked. At that time called «golden age» attention of many brains were directed to natural and precise sciences. Natural philosophy was developing and science was considered as a single entity. First we would like to mention Leonardo da Vinci, who was a genius involved at the same time into many activities: a.o. painting, sculpture, architecture, engineering, medicine and fortification. A lot of time Leonardo spent in optical technology, e.g. mirror production. $\mathrm{He}$ created special equipment to produce spherical and parabolic concave mirrors. The heritage of Leonardo's engineering activity is very big, but not all of his inventions were produced. Time-to time somebody tries to reconstruct them, and it works! Leonardo applied the golden ratio in many of his works and paintings, e.g. in the painting "The Annunciation", located in the Uffizi, Florence, Italy. In this paper we apply the idea of a "golden ratio" to modern lens design.

\section{Golden ration based optical design}

In the following and without any claim to absoluteness, the possible approach and impact of the use of the golden ratio in optical systems design is tested experimentally. To that aim, a single thin meniscus lens $\mathrm{A}$ has been designed where radii are connected by golden ratio: $\mathrm{R} 2 / \mathrm{R} 1=33.87 / 20.93=$ 1.618. Lens A looks well balanced and is free of astigmatism, distortion and all high order aberrations (see Fig.1). Optical performance stability testing: Without any claim to absoluteness, Table 1 presents results of tests carried out to check if the optical performance of the golden radii ratio lens A reported in Fig.1. was a lucky shot or a position of local minimal aberrations. To that aim, two different lenses have been designed by changing R1 in such a way, that the radii ratio yields 1.718 for lens $\mathrm{B}$ and 1.818 for lens C, respectively. For better comparison, both lenses are scaled to the same $F^{\prime}=100 \mathrm{~mm}$ and same aperture as the golden radii ratio lens A. The order of aberration is determined by the degree of the coordinate in the expansion of the transverse aberration in a series. We consider 3-d and 5-th order 
of aberrations, and we see that 5th order aberrations are almost zero because meniscus lens like this is in optimum shape and does not get high order aberrations. Third order aberrations of the meniscus with $\mathrm{R} 2 / \mathrm{R} 1=1.618$ (the golden ratio) are better than for lens B and lens C. While, in comparison to the golden radii ratio lens $\mathrm{A}$, the absolute differences in levels of aberrations of lenses $\mathrm{B}$ an $\mathrm{C}$ are not so big, the golden radii ratio lens features a clearly better performance confirming that golden ratio works...in this case.

\section{Conclusions}

In nature, beauty is often to be equivalent with being strong in survival skills, reproduction and protection. The perception of beauty herself can be found in the golden ratio which mathematically describes humans evolutionary perception of the "best solution" while interacting as a being within nature. Therefore, we might define our inclination towards beauty as an evolutionary "optimization method generated by nature". This paper demonstrated that it is worthwhile going back in time, challenging modern methodology of research methods by analyzing solutions generated by nature herself. Without any claim to absoluteness, first steps along this trail have been made experimentally and promising insights have been generated applying the "golden ratio approach" to State-of-the-Art optical systems design. The golden ratio works for the reported lens A design: if radii of a meniscus lens are connected with golden ratio number $(1,618)$; this lens has no astigmatism, distortion or all monochromatic high order aberrations and a clearly better performance than lenses featuring radius ratios located next to the golden ratio. Although the golden ratio approach to optical design reported in this paper generated impressive preliminary results, we must of course take into account that all "optimization techniques" installed by nature do not contain the claim to absoluteness but to evolutionary trial and error survival of the best. The golden ratio approach is in authors conviction a perfect complementary method to the application of modern high technology optical design software tools which are based on Snellius law, only. So it is true, that by looking back into the past we gain insights that might help us to design an optimum future; even in optical technologies

\section{References}

[1] U.Östlund, L.Kidd, Y.Wengström, N.Rowa-Dewar, "Combining qualitative and quantitative research within mixed method research designs: A methodological review", International Journal of Nursing Studies, Volume 48, Issue 3, 2011, ISSN 0020-7489,

[2] Williams, C. (2007). Research Methods. Journal of Business \& Economics Research (JBER), 5(3),

[3] O.Faehnle, "Process optimization in optical fabrication", SPIE Journal on Optical Engineering, doi:10.1117/1.OE.55.3.035106., 2016

[4] Z.Mehrjerdi, (2011), "Six-Sigma: methodology, tools and its future", Assembly Automation, Vol. 31 No. 1, pp. 79-88.

[5] Euclid; sketch and explanation of the golden ratio, book2 proposition5, Oxyrhynchus Papyri, Egypt $\left(28^{\circ} 32^{\prime} \mathrm{N}\right.$ $\left.30^{\circ} 40^{\prime} \mathrm{E}\right)$; ca. $100 \mathrm{AD}$.

[6] Francesco Melzi, "Leonardo da Vinci's "Treatise on Painting", 1632, France.

Table 1. testing optical performance stability of the golden radii ratio lens $\mathbf{A}$ in comparison to lens $\mathrm{B}$ and lens $\mathrm{C}$

\begin{tabular}{|l|c|c|c|c|c|c|c|c|c|}
\hline $\mathbf{N}$ & $\mathbf{R 2} / \mathbf{R} 1$ & $\mathbf{S A 3}$ & $\mathbf{C O 3}$ & $\mathbf{T I 3}$ & $\mathbf{S I 3}$ & $\mathbf{P E T Z}$ & $\mathbf{D I 3}$ & S'Fm m & F'mm \\
\hline $\mathbf{1}$ & $20.93 / 33.87=1.618$ & -0.079 & 0.037 & -0.0385 & -0.0385 & -0.0385 & 0.0062 & 79.12 & 100.0 \\
\hline $\mathbf{2}$ & $22.27 / 37.77=1.718$ & -0.08 & 0.039 & -0.047 & -0.044 & -0.042 & 0.0069 & 77.93 & 100.0 \\
\hline $\mathbf{3}$ & $24.17 / 43.95=1.818$ & -0.08 & 0.037 & -0.060 & -0.050 & -0.045 & 0.0077 & 76.76 & 100.0 \\
\hline
\end{tabular}

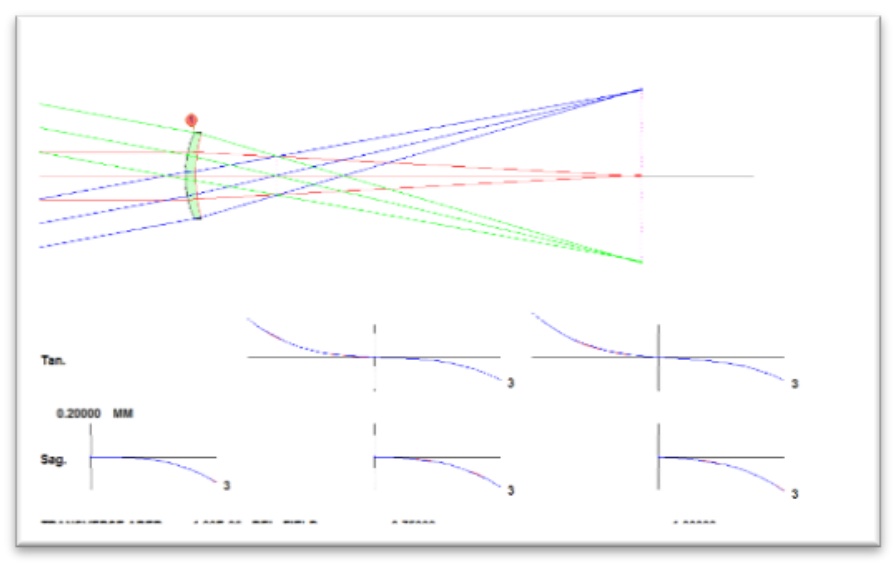

Fig. 1. Optical scheme of meniscus under goldenratio and plots of its transverse aberrations 